\title{
HIV Does Not Replicate in Naive CD4 T Cells Stimulated with CD3/CD28
}

\author{
Mario Roederer, Paul A. Raju, Dipendra K. Mitra, Leonore A. Herzenberg, and Leonard A. Herzenberg
}

Department of Genetics, Stanford University, Stanford, California 94305-5125

\begin{abstract}
In this report, we demonstrate that the $T$ cell tropic strain of HIV, LAI, does not replicate in naive CD4 $\mathrm{T}$ cells stimulated by cross-linking CD3 and CD28. In contrast, LAI replicates well in memory CD4 $\mathrm{T}$ cells stimulated in the same way. Unlike this physiologically relevant stimulation, PHA stimulates productive LAI replication in both naive and memory $T$ cells. These studies were conducted with highly purified (FACS ${ }^{\circledR}$-isolated) subsets of CD4 T cells identified by expression of both CD45RA and CD62L. Remixing of purified $T$ cells showed that naive $T$ cells do not suppress LAI replication in memory $T$ cells and that memory $T$ cells do not restore LAI expression in naive T cells. The suppression of productive LAI replication in naive $T$ cells is not due to differential expression of viral coreceptors, nor is it due to inhibition of activation of the important HIV transcription factors, nuclear factor- $\kappa \mathrm{B}$ and activator protein-1. The inherent resistance of naive $T$ cells to productive HIV infection, coupled with their proliferative advantage as demonstrated here, provides a sound basis for proposed clinical therapies using ex vivo expansion and reinfusion of CD4 T cells from HIV-infected adults. (J. Clin. Invest. 1997. 99: 1555-1564.) Key words: nuclear factor- $\kappa \mathrm{B} \cdot \mathrm{T}$ cell proliferation - fluorescence-activated cell sorter • human immunodeficiency coreceptors $\bullet$ stromal-derived factor-1
\end{abstract}

\section{Introduction}

The replication of HIV in CD4 T cells is tightly linked to the proliferation of the cells. Mitogenic stimulation is necessary for productive infection of the cells. Indeed, greater $\mathrm{T}$ cell proliferation is generally believed to lead to greater viral replication. This is the case when bulk populations of T cells (such as those found in unseparated PBMC) are infected with HIV. However, as we show in this report, this paradigm breaks down when purified subsets of $\mathrm{T}$ cells are studied.

$\mathrm{T}$ cells in PBMC are extremely heterogeneous, both functionally and phenotypically. They can be broadly divided into "naive" and "memory" subsets: naive are those cells that have not yet encountered their cognate antigen, and memory cells are those that have previously undergone an antigen-driven

Address correspondence to Mario Roederer, Beckman B011, Department of Genetics, Stanford University, Stanford, CA 94305-5125. Phone: 415-723-7149; FAX: 415-725-8564; E-mail: roederer@darwin. stanford.edu

Received for publication 6 September 1996 and accepted in revised form 19 December 1996.

J. Clin. Invest.

(C) The American Society for Clinical Investigation, Inc.

0021-9738/97/04/1555/10 \$2.00

Volume 99, Number 7, April 1997, 1555-1564 expansion (1). Naive T cells are phenotypically identified as coexpressing CD45RA and CD62L, while lacking expression of CD45RO, activation markers, and only poorly expressing CD11a (2, 3). Functionally, naive T cells are also distinct from memory cells: they respond to mitogenic stimuli with a greater calcium flux, greater proliferation, and a much more restricted cytokine profile.

Naive T cells have previously been shown to be mildly resistant to HIV infection in vitro and to harbor less virus in infected patients in vivo (4-6). However, these studies used only CD45RA or CD45RO to distinguish naive from memory T cells. While this phenotyping is relatively specific, it is not adequate for functional studies such as those presented here. In healthy individuals, on average, $5 \%$ of memory CD $4 \mathrm{~T}$ cells express CD45RA but not CD45RO, as evidenced by the lack of expression of $\mathrm{CD} 62 \mathrm{~L}$ and the coexpression of other memory phenotype markers such as CD57. This percentage increases significantly during the progression of HIV disease, such that in advanced stage patients, a vast majority of CD45RA+ CD4 $\mathrm{T}$ cells are memory $\mathrm{T}$ cells (7).

On average, half of the peripheral T cells in a healthy adult are naive. Therefore, using only CD45RA (or CD45RO) to isolate naive $\mathrm{T}$ cells will lead to an average contamination with memory $\mathrm{T}$ cells of at least $10 \%$. Since memory $\mathrm{T}$ cells are very different functionally from naive $\mathrm{T}$ cells, such a large contamination could lead to the inappropriate assignment of memoryderived cell functions to the naive T cells. Therefore, we use three-color FACS ${ }^{\circledR}$ to uniquely identify and isolate naive T cells based on the combined phenotype of CD4, CD45RA, and CD62L, resulting in highly pure, homogeneous cell populations.

In the present study, we show that there is an important difference in the ability of naive and memory $\mathrm{T}$ cells to support HIV replication, a difference far greater than had been recognized previously. In response to cross-linking of CD3 and CD28, which mimics physiological signals, naive $\mathrm{T}$ cells completely suppress HIV replication while memory T cells support active replication. Indeed, in purified $\mathrm{T}$ cells, there is little correlation between the proliferation of the cells and the replication of HIV in those cells. Our results are particularly important for the design and implementation of ex vivo CD4 $\mathrm{T}$ cell expansion for cell-based therapy of HIV disease (8).

\section{Methods}

Standard conditions. Cells were cultured in a humidified $\mathrm{CO}_{2}$ incubator at $37^{\circ} \mathrm{C}$. Cells were cultured in RPMI-1640 (Gibco Laboratories, Grand Island, NY) supplemented with $20 \%$ fetal calf serum (Gemini Bioproducts, Inc., Calabasas, CA), L-glutamine $(290 \mu \mathrm{g} / \mathrm{ml})$, penicillin $(100 \mathrm{U} / \mathrm{ml})$, streptomycin $(70 \mu \mathrm{g} / \mathrm{ml})$, and recombinant IL-2 $(25 \mathrm{U} / \mathrm{ml})$ (complete RPMI). P815/B7 (kind gift of Lewis Lanier, DNAX, Palo Alto, CA) cells were cultured as described (9). PHA (Sigma Chemical Co., St. Louis, MO) was used at $2 \mu \mathrm{g} / \mathrm{ml}$, and Leu4 (anti-CD3 monoclonal antibody; Becton Dickinson \& Co., San Jose, CA) at 50 $\mathrm{ng} / \mathrm{ml}$. For coculture, P815/B7 cells were fixed in $0.5 \%$ paraformaldehyde for 10 min and washed extensively with RPMI. Fixed P815/B7 
were added to a final concentration of $10^{5}$ cells $/ \mathrm{ml}$ to cell cultures. Control experiments were performed with fixed P815 cells (that do not express the $\mathrm{B} 7$ antigen) to prove that the fixed cells contributed no signaling other than the $\mathrm{B} 7 / \mathrm{CD} 28$ interaction and the cross-linking of soluble Leu4.

Isolation of PBMC. All human peripheral blood cells were obtained from Ficol-separated buffy coats obtained from HIV- and hepatitis-seronegative anonymous donors at the Stanford Blood Bank. After density centrifugation, PBMCs were washed and resuspended at $3 \times 10^{6}$ cells $/ \mathrm{ml}$ in complete RPMI at $37^{\circ} \mathrm{C}$ for $1 \mathrm{~h}$. Nonadherent cells were recovered and between 1 and $2 \times 10^{8}$ cells were stained with antibodies for sorting.

Cell staining, flow sorting, and analysis. Cells were stained with FITC CD62L, PE CD45RA, Cy5PE CD4, or Cy5PE CD8 (PharMingen, San Diego, CA) at room temperature, washed twice with room temperature medium, and sorted by FACS ${ }^{\circledR}$ (Becton Dickinson \& Co.). In general, at least $10^{6}$ cells of a particular phenotype were sorted. For subsequent reanalyses, cells were stained on ice. For Hoechst 33342 staining (10), cells were resuspended in medium containing $2 \mu \mathrm{g} / \mathrm{ml}$ Hoechst 33342 at $37^{\circ} \mathrm{C}$ for $7 \mathrm{~min}$. Staining was stopped by the addition of $10 \mathrm{vol}$ cold medium and the cells were washed, and then stained for antibodies. Before reanalysis, cells were washed and then fixed with $0.5 \%$ paraformaldehyde. In general, 30,000 events were collected for reanalysis. A FACStarPlus ${ }^{\circledR}$ (Becton Dickinson \& Co.) equipped with two argon ion lasers (488 and 351 $\mathrm{nm}$ excitation) was used for sorting and analysis.

Acute HIV infection. $24 \mathrm{~h}$ before infection, $5 \times 10^{6} \mathrm{ACH}-2$ cells (a T cell line chronically and productively infected with an LAI HIV isolate, reference 11) in $10 \mathrm{ml}$ of medium were stimulated with TNF $\alpha$, $1 \mathrm{ng} / \mathrm{ml}$, and PMA, $2 \mathrm{ng} / \mathrm{ml}$ for $1 \mathrm{~h}$ (12). The ACH-2 cells were then washed twice to remove residual stimulants and cultured for $23 \mathrm{~h}$. Supernatants were assayed for residual PMA and TNF $\alpha$ to assure complete removal of these stimulants. Cells to be infected were incubated with the ACH-2 supernatant, $500 \mu l$ per $10^{6}$ cells (approximate multiplicity of infection of 3), and polybrene ( $3 \mu \mathrm{g} / \mathrm{ml}$; Sigma Chemical Co.) for $30 \mathrm{~min}$ at $37^{\circ} \mathrm{C}$. Cells were washed three times and cultured at $4 \times 10^{5}$ cells $/ \mathrm{ml}$ in appropriately supplemented RPMI. Infection was monitored at various time points by removing $50 \mu \mathrm{l}$ of supernatant as assaying for p24 antigen, using an enzyme-linked immunoadsorbent assay (Abbott Laboratories Diagnostic Division, Chicago, IL). For each experiment, a standard curve was generated for known concentrations of p24 to determine the absolute concentration in samples.

Semiquantitative PCR for CXC chemokine receptor 4 and CC chemokine receptor 5. RNA from 250,000 sorted cells (naive or memory) was isolated using RNAzol B (TelTest, Inc., Friendswood, $\mathrm{TX})$. Total cDNA was synthesized using $1 \mu \mathrm{g}$ random hexamers in a $35-\mu l$ reaction volume. $3 \mu \mathrm{l}$ of each cDNA was then amplified by PCR using primers specific for each gene. An aliquot of each PCR reaction was removed after 25, 30, 35, and 40 cycles. Products were separated on a $1 \%$ agarose gel and visualized by ethidium bromide staining. Quantitation was performed on digital images of the gel; results are presented from aliquots taken while the amplification was still proceeding exponentially. The following primer pairs were used: $\beta$-actin, TGACGGGGTCACCCACACTGTGCCCATCTA and CTAGAAGCATTGCGGTGGACGATGGAGGG; CXC chemokine receptor 4 (CXCR-4) ${ }^{1}$, GCAGCAGGTAGCAAAGTGAC and CGTTCCACGGGAATGGAGAG; CC chemokine receptor 5 (CCR-5), CAAGCTGCTTCTGGTTGGGC and GCCTCAGCCCAGGTGAACCC.

Chemokine-induced calcium flux. Naive and memory T cells were sorted and cultured for $24 \mathrm{~h}$ in IL-2 under standard conditions. Cells

1. Abbreviations used in this paper: AP-1, activator protein-1; CCR-5, "CC" chemokine receptor 5; CXCR-4, "CXC" chemokine receptor 4; LTR, long terminal repeat; NF-кB, nuclear factor-кB; SDF, stromalderived factor. were centrifuged and resuspended in $1 \mathrm{ml}$ of growth medium at room temperature. Indo-1 (Molecular Probes, Inc., Eugene, OR) was added to a final concentration of $3 \mu \mathrm{M}$; cells were incubated for 30 min. Cells were centrifuged and resuspended at a concentration of $10^{7}$ cells $/ \mathrm{ml}$ in "load" buffer (HBSS supplemented with $1 \%$ fetal calf serum). For each sample condition, $10^{6}$ cells were diluted to a final volume of $2 \mathrm{ml}$ in "flux" buffer (HBSS supplemented with $1.6 \mathrm{mM}$ $\mathrm{CaCl}_{2}, 5 \mathrm{mM} \mathrm{MgCl}_{2}, 25 \mathrm{mM}$ Hepes, $\mathrm{pH} 7.3$ ), and placed in a fluorometry cuvette with a magnetic stir bar. Indo- 1 fluorescence was continuously monitored at two wavelengths (400 and $490 \mathrm{~nm}$ ); the ratio is monotonically related to calcium concentration. Highly purified, recombinant SDF-1 (R \& D Systems, Inc., Minneapolis, MN) was added to a final concentration of $250 \mathrm{pM}$ after collection of resting Indo-1 fluorescence. Collection was continued for several more minutes.

Proliferative responses. Bulk PBMCs and sorted subpopulations (either HIV- or mock-infected) were cultured in 200- $\mu$ l volumes of a 96-well plate in triplicate $\left(10^{4}\right.$ cells/well). $3 \mathrm{~d}$ after stimulation, $1 \mu \mathrm{Ci}$ of ${ }^{3} \mathrm{H}$-thymidine (Amersham Corp., Arlington Heights, IL) was added to the cultures. $24 \mathrm{~h}$ later, incorporated radioactivity was determined using an automated harvesting and counting system.

Quantitative competitive PCR for cytokine expression. mRNA for IL-2, IL-4, and $\gamma$-IFN were quantitated by quantitative competitive PCR (13); the method will be presented in detail elsewhere (Mitra, D., J. Tung, L.A. Herzenberg, L.A. Herzenberg, and M. Roederer, manuscript in preparation). Briefly, the competitor in each case was an artificially constructed RNA containing the precise cytokine mRNA sequence but with an internal deletion. These were constructed by PCR amplification using a $5^{\prime}$ primer that is homologous to two portions of each gene 69,85 , and 87 bp apart (for IL-2, IL-4, and $\gamma$-IFN, respectively), together with a $3^{\prime}$ primer near the terminus of the gene. The $5^{\prime}$ primer also contains the T7 RNA polymerase binding site. In the sequences given below, underlined regions are the $\mathrm{T} 7$ binding site, and the asterisk $\left(-*_{-}\right)$marks the position where the primer bridges a deletion in the mRNA sequence of the cytokine. Primers used were: IL-2, TAATACGACTCACTATAGGGATGTACAGGATGCAACTCCTGTCT-*-CTACAACTGGAGCATTTACTGCTG $\left(5^{\prime}\right)$ and GTATCTATGTAGATTGGTTAG (3'); IL-4, TAATACGACTCACTATAGGG-ATGGGTTCTCACCTCCCAACTGCT-*-CTGTGCACCGAGTTGACC (5') and CCTGTGAAGGAAGCCAACCAGAGT (3'); $\gamma$-IFN, TAATACGACTCACTATAGGG-ATGAAATATACAAGTTATATCTTGGCTTT-*-GCAGGTCATTCAGATGTAGCGGATAATG $\left(5^{\prime}\right)$ and CTAAGCAAGATCCCATGGGTTGTGTGT (3'). These primers were used to amplify total cDNA from PHA-stimulated PBMC. The product bands were gel purified and used as substrates for in vitro RNA synthesis. The resulting full length RNA was quantitated spectrophotometrically and stored in aliquots at a known number of molecules per microliter.

To determine the mRNA messages from the sorted naive and memory cells, $10^{5}$ cells were stimulated and mRNA was isolated as above. Seven aliquots, each containing $10 \%$ of the total mRNA, were mixed with known amounts of each cytokine deletion transcript (half$\log$ dilutions starting at $1.3 \times 10^{6}$ copies of $\Delta \mathrm{IL}-2,10^{5}$ copies of $\Delta \mathrm{IL}-4$, and $10^{6}$ copies of $\Delta \gamma$-IFN). cDNA from each mixture was synthesized as above. An aliquot of each cDNA was then amplified by PCR using nested primers specific for each cytokine message. Products were separated on a $1 \%$ agarose gel and visualized by ethidium bromide staining. Quantitation of the wild-type mRNA (cell derived) and deleted RNA (standard) were performed by image analysis and the ratio of the size-corrected intensities were recorded (13). These values were used to compute the number of molecules of each mRNA per cell. The following nested primers were used: IL-2, ATGTACAGGATGCAACTCCTGTCT and TCAAAGCATCATCTCAACACTAAC, IL-4, ATGGGTTCTCACCTCCCAACTGCT and CCTGTGAAGGAAGCCAACCAGAGT, $\gamma$-IFN, ATGAAATATACAAGTTAT ATCTTGGCTTT and ATGCTGTTTCGAGGTCGAAGAGCATC. The amplified product sizes for each gene and its deletion construct are: IL-2, 460 and 391 bp; IL-4, 383 and 278 bp; $\gamma$-IFN, 496 and 409 bp. 
Quantitative PCR for HIV DNA and/or RNA was performed as previously described (14). For these experiments, naive and memory cells were sorted, cultured, and infected with HIV as described above. $20 \mathrm{~h}$ after infection, cells were centrifuged, washed three times with growth medium, and frozen on dry ice. Cell pellets were later dissolved and assayed as described (14). As a control, cells were incubated with the HIV-containing supernatant and immediately washed and frozen. These samples had $<20 \%$ of the signal from the 20 -h cultures.

HIV long terminal repeat lac Z reporter cell assay. The development, properties, and application of the 293.27 .2 cell line have been published (15). Support medium for experiments and maintenance of cells was Dulbecco's modified Eagle's medium (Gibco Laboratories). For a typical experiment, 2,000 cells per well were plated $12-24 \mathrm{~h}$ before the experiment. Supernatants from cultures were applied to the cells for 6 to $8 \mathrm{~h}$. $\beta$-Galactosidase activity was measured using 4-methylumbelliferyl- $\beta$-D-galactosidase (MUG) as previously described (16).

Electrophoretic mobility gel shift assays. Cells were stimulated for $6 \mathrm{~h}$, and then harvested and centrifuged $\left(10 \mathrm{~min}, 1,200 \mathrm{rpm}, 4^{\circ} \mathrm{C}\right)$, washed in $1 \mathrm{ml}$ ice cold Tris-buffered saline and spun $15 \mathrm{~s}, 14,000 \mathrm{rpm}$, $4^{\circ} \mathrm{C}$, in an Eppendorf centrifuge. Cells were washed once in $0.4 \mathrm{ml}$ buffer A (10 mM Hepes, pH 7.8, $10 \mathrm{mM} \mathrm{KCl,} 2 \mathrm{mM} \mathrm{MgCl}_{2}, 1 \mathrm{mM}$ DTT, $0.1 \mathrm{mM}$ EDTA, $0.1 \mathrm{mM}$ PMSF), supplemented with the protease inhibitors Antipain ( $1 \mathrm{mg} / \mathrm{ml}$, Sigma Chemical Co.) and Leupeptin $(0.3 \mathrm{mg} / \mathrm{ml}$, Sigma Chemical Co.), and incubated on ice for 15 min. $25 \mu$ l of a $10 \%$ NP-40 solution (Sigma Chemical Co.) was added and cells were vigorously vortexed for $15 \mathrm{~s}$, and then centrifuged $(30 \mathrm{~s}$, $14,000 \mathrm{rpm})$. Pelleted nuclei were resuspended in $50 \mu \mathrm{l}$ buffer $\mathrm{C}(50 \mathrm{mM}$
Hepes, pH 7.8, $50 \mathrm{mM} \mathrm{KCl}, 300 \mathrm{mM} \mathrm{NaCl}, 0.1 \mathrm{mM}$ EDTA, $1 \mathrm{mM}$ DTT, $0.1 \mathrm{mM}$ PMSF, $10 \% \mathrm{vol} / \mathrm{vol}$ glycerol). After gentle mixing for $20 \mathrm{~min}$, the tubes were spun for $5 \mathrm{~min}$ at $14,000 \mathrm{rpm}$, after which the supernatant containing the nuclear proteins was transferred to another tube. Protein samples were stored at $-70^{\circ} \mathrm{C}$. Mobility shift (gel retardation) assays were done essentially as described $(17,18)$. The binding reactions were carried out with $10 \mu \mathrm{g}$ of protein. Oligonucleotides used in these studies are ${ }^{32} \mathrm{P}$-labeled monomers of the binding

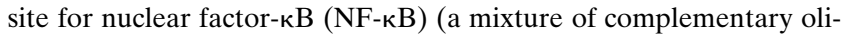
gonucleotides with four base $5^{\prime}$-overhangs, TCGAGTCAGAGGGGACTTTCCGAG and CGACTCGGAAAGTCCCCTCTGAC) or activator protein-1 (AP-1) (TCGAGTGACTCAGCGCG and TCGACGCGCTGAGTCAC).

\section{Results}

HIV infection of purified CD4 T cell subsets. Subsets of CD4 T cells from healthy adult donors were sorted to $>98 \%$ purity by FACS $^{\circledR}$ sorting ( $>99 \%$ expressed CD4). These subsets were identified by the $\mathrm{FACS}^{\circledR}$ on the basis of five different parameters (Fig. $1 A$ ): forward and side-scattered light (identifying lymphocytes), and the expression of CD4, CD45RA, and CD62L. This combination of markers is necessary to uniquely identify naive $\mathrm{T}$ cells (1-3): in healthy donors, there is a variable representation (average 5\%) of memory CD4 T cells that express CD45RA (and not CD45RO) but do not express
A. Sort
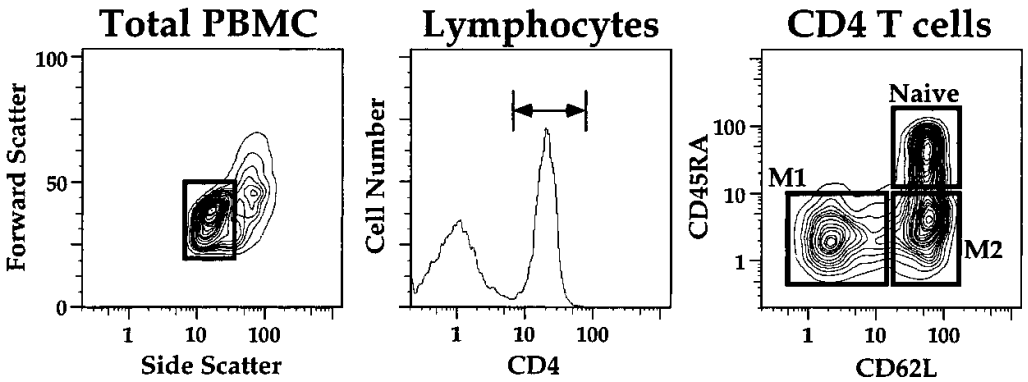

\section{B. Reanalysis after 36h \\ Before infection or stimulation}

\section{Reanalysis after $6 \mathrm{~d}$ \\ HIV-infected, $\mathrm{CD} 3 / \mathrm{CD} 28$ stimulated}
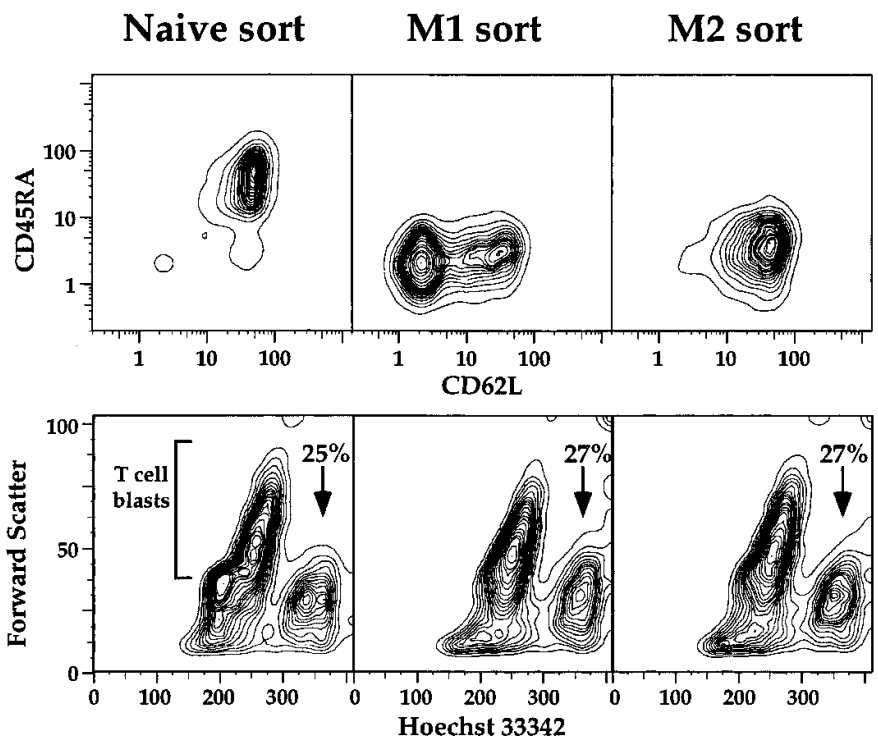

Figure 1. FACS ${ }^{\circledR}$ analysis and sorting of $\mathrm{CD} 4$ subpopulations. $(A)$ For sorting of CD4 subsets, total PBMC were stained for expression of CD62L, CD45RA, and CD4. Sorting gates are shown, (left) lymphocytes, (middle) CD4-positive T cells, (right) naive, memory M1, and memory M2 cells. Cell purities were always assessed immediately after sorting and were always $>98 \%$. (B) After $36 \mathrm{~h}$ of culture without stimulation, cells were restained. The graphs are shown "ungated;" i.e., all events are displayed. The cells were uniformly CD4 positive and retained the sorting phenotype. A fraction of the M1 cells are expressing low levels of CD62L. This may be due either to an upregulation of the gene, or more likely to inadequate resolution of the two populations at the time of sorting (note in the top right panel of $A$ that there is a continuum of expression of CD62L between the M1 and M2 populations). (C) Cells were analyzed after another $6 \mathrm{~d}$ of culture. Here, cells are stained with antibodies as well as Hoechst 33342, which reveals apoptotic cells (see also Table I). As an example, analyses of cells from the naive, M1, and M2 sorts that were infected with HIV and stimulated through the CD3/

CD28 pathways are shown. "Blasting" of the cells is indicated by the significant increase in size (forward scatter); the small (low forward scatter) brightly Hoechst-staining cells are apoptotic (numbers over the arrows indicate percentage of these apoptotic cells in the culture). 

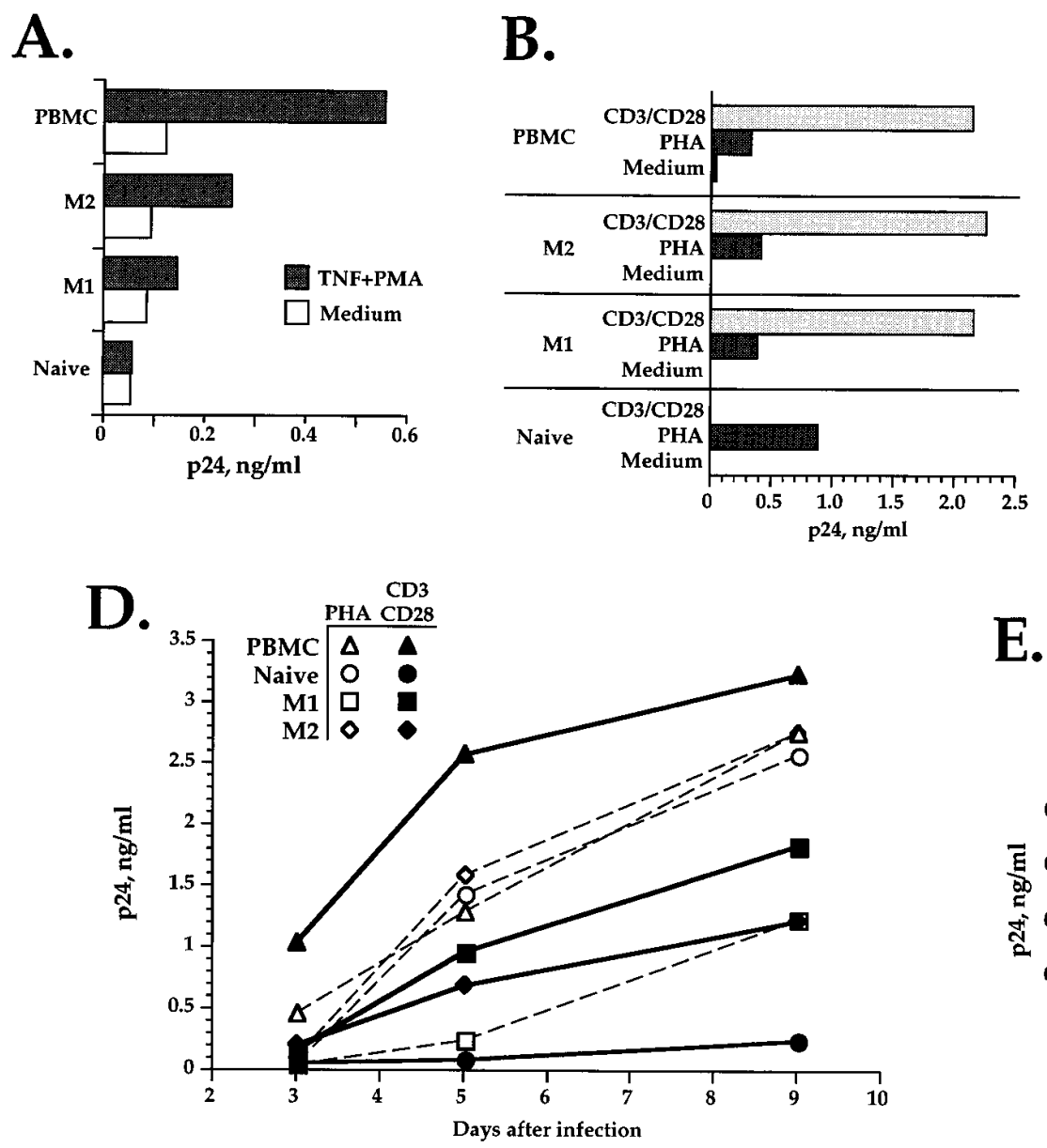

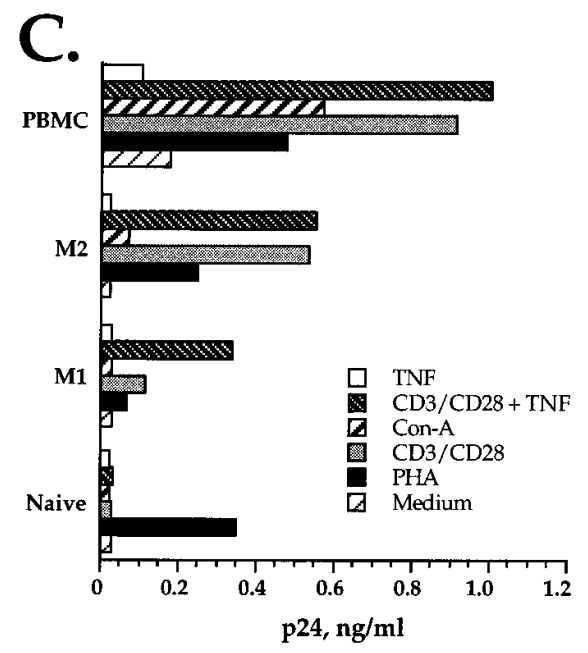

E.

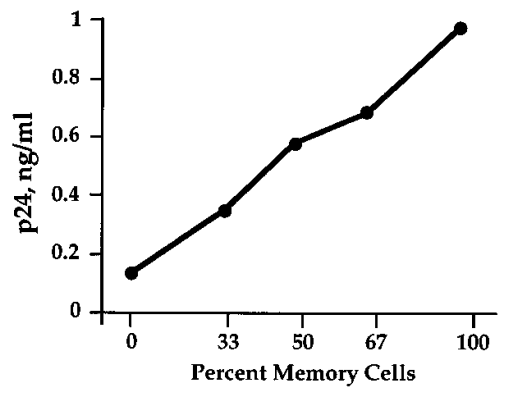

Figure 2. HIV does not replicate in CD3/CD28-stimulated naive CD4 T cells. (A) CD4 subsets were sorted to purity as shown in Fig. 1, infected with HIV, and left either unstimulated or stimulated with TNF $\alpha(1 \mathrm{ng} / \mathrm{ml})$ and PMA $(2 \mathrm{ng} / \mathrm{ml})$. After $3 \mathrm{~d}$, supernatants were assayed for soluble p24. p24 production was low in these cultures, but was significantly increased by TNF $\alpha$ and PMA stimulation in PBMC and purified memory cells; p24 in the naive supernatants was not above background (as determined, for instance, by the infection of sort-purified CD8 T cells with HIV). The amount of p24 detected in these cultures is well correlated with the amount of infectious virus present in the supernatant (30). (B) In this experiment, we infected sort-purified cells with HIV and stimulated with either PHA ( $2 \mu \mathrm{g} / \mathrm{ml})$, CD3/CD28, or left unstimulated. PHA stimulated high p24 production in all CD4 subsets and total PBMC; however, costimulation of the CD3/CD28 pathways resulted in no detectable p24 from naive T cells above background. For each cell type, cells were infected before separation into different wells and stimulation conditions. (C) CD4 subsets or total PBMC were infected with HIV and stimulated under the conditions shown. p24 was assayed after 5 d. Only PHA was capable of stimulating the production of p24 in infected naive T cells (ConA was used at $10 \mu \mathrm{g} / \mathrm{ml})$. TNF $\alpha$ (10 ng/ml) was unable to rescue the p24 production from naive T cells stimulated with CD3/CD28. (D) The kinetics of p24 production over 9 d is shown for PHA- or CD3/ CD28-stimulated cells. After 9 d, the amount of p24 in the naive cultures stimulated with CD3/CD28 is low but significantly above background. $(E)$ Naive and memory M1 T cells were sorted and then remixed in different percentages. Cells were then infected with HIV and stimulated with $\mathrm{CD} 3 / \mathrm{CD} 28$. The $\mathrm{p} 24$ production correlates well with the fraction of cells that are of the memory phenotype, suggesting that naive $\mathrm{T}$ cells do not produce an inhibitory factor, and that memory cells cannot "rescue" the HIV replication in naive T cells. Similar results were obtained when naive T cells were mixed with memory M2 cells or with total memory (M1 + M2) cells. Representative data from eight independent experiments on cells from 10 donors are shown.

CD62L (reference 7 and data not shown). Reanalysis of sorted CD4 subpopulations was performed immediately after the sort to verify that the purity was in excess of $98 \%$.

Purified T cells were then cultured for $24-36 \mathrm{~h}$ in the absence of stimulation. The methods we use for staining cells with fluorescently conjugated antibodies and the subsequent sorting are done in such a way that the functional capacity of these cells is unaltered (19). The phenotype of the sorted populations was essentially unaltered after this culture phase (Fig. $1 B$ ).
Purified T cells or bulk PBMC were then infected with HIV derived from the chronically infected cell line ACH-2, the LAI strain (11). Cells were incubated in the presence of HIV for $2 \mathrm{~h}$ and washed extensively. After the infection, cells were divided into parallel cultures and stimulated under different conditions; thus, in any particular experiment, all cells of a particular phenotype have been identically infected with HIV.

We then stimulated the infected cells with PHA, a lectin that has been routinely used to generate productive HIV infection in human cells. PHA stimulated significant HIV replica- 
tion in all purified CD4 subsets, as well as bulk PBMC (Fig. 2). Indeed, naive T cells produced as much HIV on a per-cell basis as did memory cells, in agreement with previous reports (4). Thus, the infection was successful in all subsets.

CD3/CD28-stimulated naive $T$ cells suppress productive HIV infection. We also stimulated cells through the CD3/ CD28 pathways using the cell line P815/B7 (9). This murine mastocytoma is transfected to express the ligand for CD28; in addition, it expresses murine $\mathrm{Fc}$ receptors that are capable of binding and cross-linking soluble antibody (i.e., Leu4, which is anti-CD3). This method provides a substantially physiological approach to cross-linking the CD3 and CD28 molecules. Nearly $100 \%$ of CD4 T cells in healthy adults express CD28.

Surprisingly, although CD3/CD28 stimulation led to significantly increased HIV replication in the preinfected PBMC and memory CD4 T cells (Fig. 2), naive T cells produced no detectable virus. The naive $\mathrm{T}$ cells were clearly infected with HIV, since, as indicated above, parallel cultures of naive cells stimulated with PHA produced significant virus. (Note that in these experiments, purified subsets were infected before separation into different culture conditions.) Thus, stimulation by crosslinking CD3 and CD28, which is a powerful mitogenic stimulation for naive $\mathrm{T}$ cells (reference 8; see below), suppressed productive HIV replication in naive T cells.

TNF $\alpha$ and PMA are powerful stimulants of the HIV long terminal repeat (LTR) and HIV replication in PHA-blasted PBMC (15). However, TNF $\alpha$ and PMA stimulation, which is not mitogenic, leads to a significant augmentation of the low but clearly detectable HIV replication in bulk PBMC and purified memory T cells (Fig. $2 \mathrm{~A}$ ). In contrast, naive T cells produced no detectable virus when either unstimulated or stimulated with the combination of TNF $\alpha$ and PMA (p24 levels in these cultures were not above background levels obtained by HIV "infection" of purified CD8 T cells). The lack of enhancement by TNF $\alpha$ and PMA was not due to a failure of HIV to infect the naive $T$ cells nor due to a failure of the cells to activate the necessary nuclear transcription factors (see below).

Similarly, the addition of TNF $\alpha$, which has been shown to increase HIV LTR-directed transcription through the activation of NF-кB (20) did not "rescue" viral production in the CD3/CD28-stimulated naive T cells. TNF $\alpha$ did potentiate CD3/CD28-stimulated HIV replication mildly in PBMC and memory cells.

In general, the kinetics of viral production after infection of the cells is consistent with many previous publications (Fig. $2 D$ ). Little or no virus is detectable in purified $\mathrm{T}$ cell cultures until after $2 \mathrm{~d}$ postinfection. Viral production increases over time after this. Interestingly, $9 \mathrm{~d}$ after $\mathrm{CD} 3 / \mathrm{CD} 28$ stimulation, there is a small amount of HIV detectable in the naive cultures. Therefore, the lack of HIV production in naive T cells persists for almost a week-a time frame consistent with the time required for differentiation of naive $\mathrm{T}$ cells into memory $\mathrm{T}$ cells (2).

Purified $T$ cell subsets do not cross-regulate HIV replication. We performed mixing experiments to ask whether the differential replication in CD4 subsets could be ascribed to a soluble factor. Naive and memory $\mathrm{T}$ cells were sorted to purity and then remixed at various ratios. These artificially generated mixtures were then infected with HIV. As shown in Fig. 2 E, the amount of virus production from these mixtures is closely proportional to the fraction of memory $\mathrm{T}$ cells in the cultures. This suggests that the major source of virus in infections of mixed cultures is the memory cells. In addition, it excludes the
A.
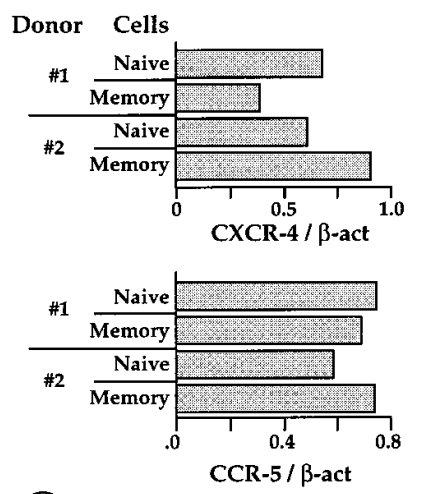

C.

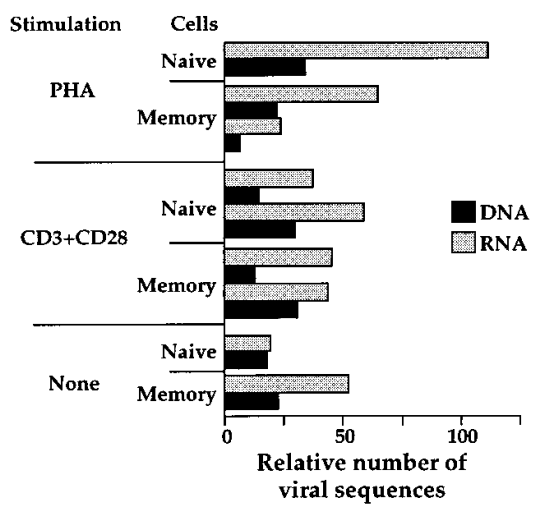

Figure 3. Similar infectivity of naive and memory T cells is consistent with coreceptor expression. (A) Naive and total memory (M1 + M2) cells were sorted, RNA was extracted and reverse transcribed. Semiquantitative PCR using primers specific for CXCR-4, CCR-5, and $\beta$-actin mRNAs was performed on aliquots of each reverse transcription. The amount of PCR product was quantitated; the expression of CXCR-4 and CCR-5 messages is expressed relative to the $\beta$-actin expression (there was less than twofold variation in $\beta$-actin mRNA between samples). (B) Sorted naive and memory cells were stimulated with purified SDF-1, the ligand for CXCR-4 $(27,28)$. Cytoplasmic calcium concentration is shown as a function of time. SDF-1 was added at $10 \mathrm{~s}$ to a final concentration of $250 \mathrm{pM}$ (arrow). Quantitative differences in calcium mobilization may reflect differential signal transduction mechanisms, similar to differences observed for naive and memory T cells upon CD3 receptor engagement (19). (C) Sorted naive and memory cells were infected as in Fig. 2. After $20 \mathrm{~h}$, cells were assayed for viral DNA, or viral DNA+RNA sequences using quantitative PCR (14) to assess the amount of viral penetration into the cells. The relative amount of viral sequence is shown for the different culture stimulations. A second experiment on cells from a different donor is shown for some stimulations. Detection of viral DNA does not necessarily reflect viral integration, but quantitates the extent of reverse transcription that has occurred. (Control cultures analyzed immediately after exposure to the infectious supernatant had $<20 \%$ of the signal found in the 20 -h experiment.)

possibility that naive $\mathrm{T}$ cells produce a soluble factor that inhibits HIV replication. Finally, data from the mixing experiment suggests that memory $\mathrm{T}$ cells do not produce a factor that is absolutely required for HIV replication in T cells.

Expression of HIV coreceptors in CD4 T cell subsets. Differential infectivity of the purified $\mathrm{T}$ cells could account for some differences in HIV production. While naive T cells have been shown to be approximately fivefold less infectable than mem- 
ory T cells (4), we found little difference in the production of HIV after PHA stimulation. Nonetheless, it is possible that PHA mediates viral fusion in the absence of viral coreceptors.

Recently, two different surface molecules on $\mathrm{T}$ cells required for entry of HIV (other than CD4), CXCR-4/fusin (21) and CCR-5 (22-26) have been identified. To assess the potential role of these molecules in HIV infection of purified CD4 subsets, we quantitated expression of the messenger RNA molecules encoding these gene products. Using semiquantitative PCR, we found that both naive and memory T cells express similar levels of mRNA for these two coreceptors (Fig. $3 A$ ).

Consistent with this expression, preliminary studies with fluorescently conjugated antibodies to CXCR-4 show that all CD4 T cells express the receptor on the surface (M. Roederer, unpublished observations; N. Landau, personal communication). Furthermore, costimulation through CD3 and CD28 increases the expression of the CXCR- 4 gene on primary $\mathrm{T}$ cells (C. June, personal communication).

Indeed, as shown in Fig. 3 , both purified naive and memory $T$ cells exhibit a calcium flux in response to stromal-derived factor (SDF)-1, the ligand for CXCR-4 $(27,28)$. Thus, both subsets express equivalent amounts of CXCR-4 and CCR-5 message and similar levels of CXCR-4 protein, and both subsets express functional CXCR-4 capable of transducing signal. Quantitative differences in calcium flux between these subsets do not necessarily reflect similar quantitative differences in receptor expression: CD4 subsets show differential calcium flux in response to $\mathrm{CD} 3$ triggering, even though they express identical amounts of CD3 (19).

The possibility that quantitatively different surface expression of viral coreceptors on naive and memory cells leads to differential infectivity was directly assessed by quantitating the amount of viral sequences present in the cells $20 \mathrm{~h}$ postinfection. At this early time point, which would be relatively soon after potential proviral integration, there should be little effect of the stimulants on the upregulation of viral expression. Thus, quantitating the viral RNA (or viral DNA) levels should reflect primarily the amount of virus that has entered the cell (although not necessarily integrated). Indeed, quantitative PCR for either viral DNA or viral DNA plus viral RNA showed substantially equivalent levels of infection in both naive and memory cells irrespective of the culture conditions (Fig. $3 C$ ). This confirms that stimulation by $\mathrm{PHA}$ or $\mathrm{CD} 3 / \mathrm{CD} 28$ after the infection of the cells did not affect the entry of HIV into naive or memory cells.

Differential proliferation or apoptosis of T cell subsets does not account for differential HIV production. We undertook a series of functional assays on the purified CD4 subsets to try to understand mechanisms that might account for the differential ability of naive and memory cells to support productive HIV replication. The simplest assay quantitates the proliferative capacity on a per-cell basis. As is well known, naive T cells have, in general, a greater proliferative capacity (on a per-cell basis) than do their memory counterparts. As Fig. 4 shows, this is the case for both PHA stimulation as well as CD3/CD28 stimulation. Indeed, the CD3/CD28 stimulation is a very powerful mitogenic signal, resulting in manyfold greater proliferation than PHA. HIV infection of the cultures, while having a mild damping of the proliferative capacity, did not alter the relative stimulations by PHA and CD3/CD28. Despite the fact that the best proliferation observed in these various cultures was for naive $\mathrm{T}$ cells stimulated by $\mathrm{CD} 3 / \mathrm{CD} 28$, no viral production was ob-

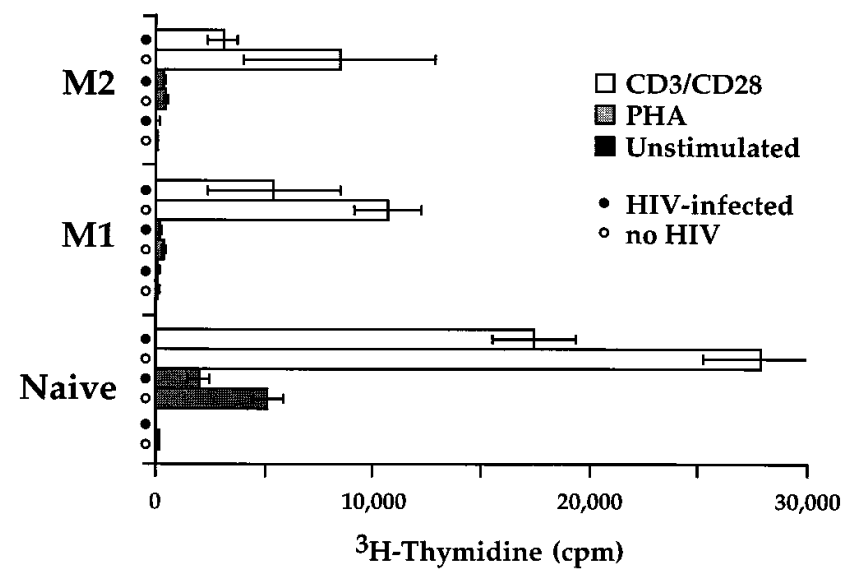

Figure 4. Naive T cells stimulated with $\mathrm{CD} 3 / \mathrm{CD} 28$ proliferate much more than memory T cells. $4 \mathrm{~d}$ after HIV infection (or mock infection), cell proliferation was assessed by the incorporation of ${ }^{3} \mathrm{H}$-thymidine. As expected, CD3/CD28 stimulation provides a much stronger proliferative signal than does PHA. Infection with HIV consistently resulted in a slight decrease in proliferation of the T cells (but no statistically significant change in apoptosis). This is one of two experiments testing proliferation on HIV-infected cells. Results from mock-infected cells are consistent with many independent experiments.

served in those cultures (some of the experiments are presented in Fig. 2 B; all proliferation experiments included p24 measurements). This observation breaks the previously accepted link between cellular and viral proliferation, and raises the question of whether other aspects of cellular activation are necessary for HIV replication; i.e., not necessarily ones that lead to proliferation.

Kobayashi et al. (29) demonstrated an increase of apoptosis in HIV-infected T cells. We assessed apoptosis $6 \mathrm{~d}$ after HIV infection (Fig. $1 C$ and Table I) for all culture conditions (PHA, CD3/CD28, and with or without HIV infection). While the extent of apoptosis depended on the stimulation condition and cell type, we found no discernible trend in apoptosis comparing infected with uninfected cultures.

Naive and memory $T$ cells have significantly different cytokine profiles. In general, naive $\mathrm{T}$ cells make only IL-2; memory $\mathrm{T}$ cells can make the entire spectrum of $\mathrm{T}$ cell cytokines. Using competitive PCR, we quantitated the production of IL-2, IL-4, and $\gamma$-IFN by the CD4 subsets. As shown in Fig. 5, the production of these cytokines is quantitatively identical for the same subset isolated from different individuals. Thus, differences in cytokine profiles for bulk populations of $\mathrm{T}$ cells are due to differences in the relative representation of functionally distinct subsets.

To assess the ability of the subsets to produce cytokines that directly stimulate HIV LTR-directed transcription, we used a reporter gene system (15) in cells that are responsive primarily to IL-1 and TNF $\alpha$. In general, supernatants from stimulated cultures showed very strong stimulatory activity for the HIV LTR, regardless of whether the cultured subsets had been infected with HIV. However, supernatants from naive cells stimulated with $\mathrm{CD} 3 / \mathrm{CD} 28$ were far less effective than were supernatants from naive T cells stimulated with PHA. In fact, supernatants from naive $\mathrm{T}$ cells stimulated with PHA were as 
Table I. Apoptosis in Purified T Cell Cultures

\begin{tabular}{|c|c|c|c|c|c|c|}
\hline \multirow[b]{2}{*}{ Stimulation } & \multicolumn{2}{|c|}{ Naive } & \multicolumn{2}{|c|}{ M 1} & \multicolumn{2}{|c|}{ M 2} \\
\hline & No HIV & $+\mathrm{HIV}$ & No HIV & $+\mathrm{HIV}$ & No HIV & $+\mathrm{HIV}$ \\
\hline \multicolumn{7}{|l|}{ Experiment 1} \\
\hline None & 42 & 55 & 14 & 13 & 6.3 & 11 \\
\hline PHA & 18 & 10 & 21 & 27 & 28 & 21 \\
\hline $\mathrm{CD} 3 / \mathrm{CD} 28$ & 47 & 40 & 32 & 31 & 24 & 25 \\
\hline \multicolumn{7}{|l|}{ Experiment 2} \\
\hline None & 30 & 46 & 7.8 & 12 & 16 & 9.7 \\
\hline PHA & 8.7 & 10 & 25 & 13 & 25 & 23 \\
\hline CD3/CD28 & 26 & 25 & 29 & 27 & 31 & 27 \\
\hline
\end{tabular}

Numbers represent the fraction of cells that were apoptotic using the Hoechst 33342 assay by FACS ${ }^{\circledR}$. Purified cells were infected with HIV or mock infected, stimulated as shown, and cultured for $6 \mathrm{~d}$.

stimulatory as those from memory cells. This difference parallels the observed difference in the capacity of HIV to productively replicate in these cultures (Fig. 2).

Supernatants from memory cells are clearly stimulatory for HIV transcription and presumably for replication. However, these soluble factors did not restore productive HIV infection in naive $\mathrm{T}$ cells stimulated with $\mathrm{CD} 3 / \mathrm{CD} 28$ when memory and naive $\mathrm{T}$ cells were mixed (Fig. 2 E). Thus, naive $\mathrm{T}$ cells appear to lack the receptors to transmit the signals from the soluble factors.

Activation of nuclear factors in naive T cells is normal. Finally, we assessed the capacity of the subsets to activate nuclear factors known to interact with regulatory regions on the HIV LTR. The most important of these is NF-kB, which has been shown to significantly enhance HIV LTR-directed transcription and is necessary and sufficient for TNF $\alpha$-mediated activation of the HIV LTR (20). As shown in Fig. 6, both NF-kB and AP- 1 are activated in stimulated naive and memory T cells. While not unexpected, this finding is surprising in view of the fact that no detectable HIV is produced by the stimulated naive $\mathrm{T}$ cells. Therefore, the block of HIV production within these cells when stimulated by CD3/CD28 does not include a block in the activation of nuclear factors important to HIV transcription regulation.

\section{Discussion}

The data we present demonstrates that HIV replication is not necessarily correlated with cellular replication. Indeed, in these experiments, the conditions that resulted in the greatest cellular replication resulted in undetectable viral production
A.

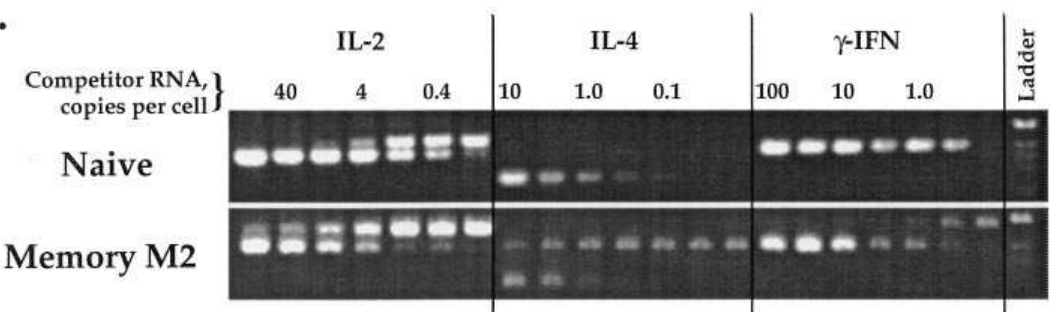

B.

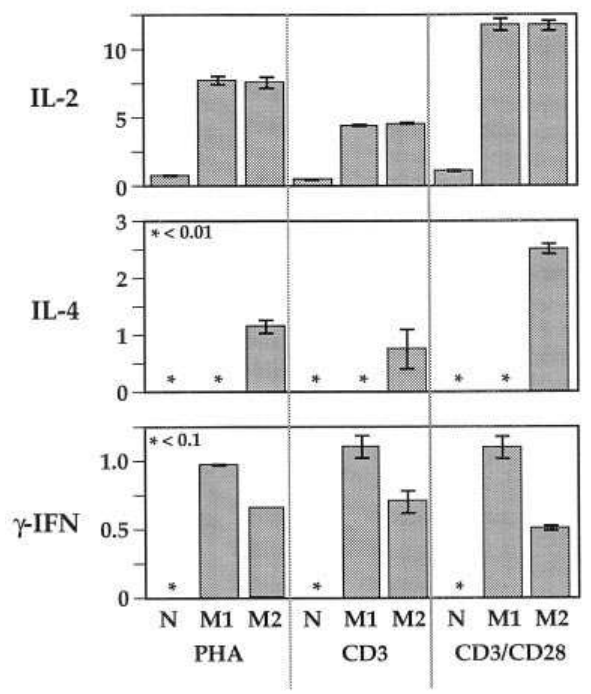

C.

Supernatant
from

PBMC

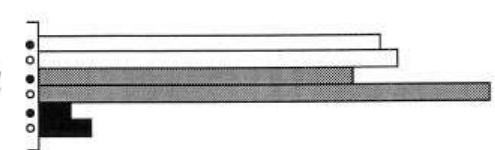

M2

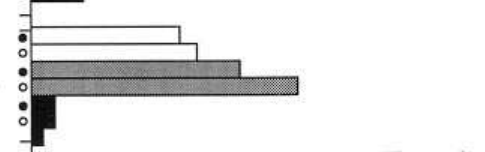

M1

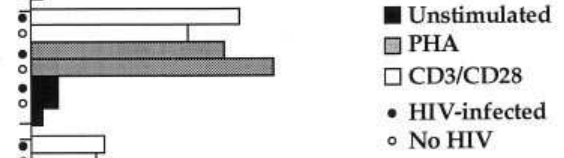

Naive

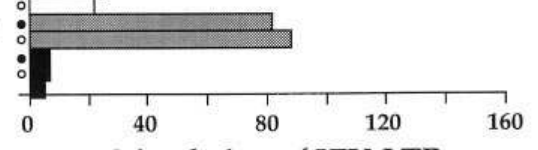

Figure 5. CD4 subsets have significantly different cytokine profiles. $(A)$ Quantitative competitive PCR (13) was performed to assess the production of IL-2, IL-4, and $\gamma$-IFN by CD4 subsets. mRNA was isolated from each cell type after stimulation for $8 \mathrm{~h}$. Aliquots of the mRNA were mixed with dilutions of known numbers of RNAs identical to each cytokine, but with an internal deletion. Shown is a typical gel for naive and memory M2 cells stimulated with CD3/CD28. (B) Quantitation of the competitive PCR assay for sorted cells from four different donors; asterisks indicate undetectable mRNA production. Cells were either stimulated with PHA, cross-linked CD3 alone, or cross-linked CD3 and CD28. Error bars are mean $\pm 1 S D$. There is a remarkable consistency in the number of cytokine messenger RNA molecules per cell (ordinate) for purified subsets of CD4 T cells. Only M2

memory cells produced IL-4, consistent with their known role of providing B cell help. $(C)$ Supernatants from HIV-infected (or mock-infected) cultures of sorted CD4 cells or PBMC were added to the 293.72.2 cell line in quadruplicate. This cell line expresses $\beta$-galactosidase under control of the HIV LTR. We have used it extensively to assess the regulation of the HIV LTR (15). The 293.27 .2 cells were cultured for $8 \mathrm{~h}$ in the presence of the supernatant, and then assayed for $\beta$-galactosidase activity. The 293.27 .2 cell line is not responsive to PHA or anti-CD3. $10 \mathrm{U}$ activity represents the basal (unstimulated) expression of $\beta$-galactosidase by the cell line. Replicate wells had an average variation of $<10 \%$. 


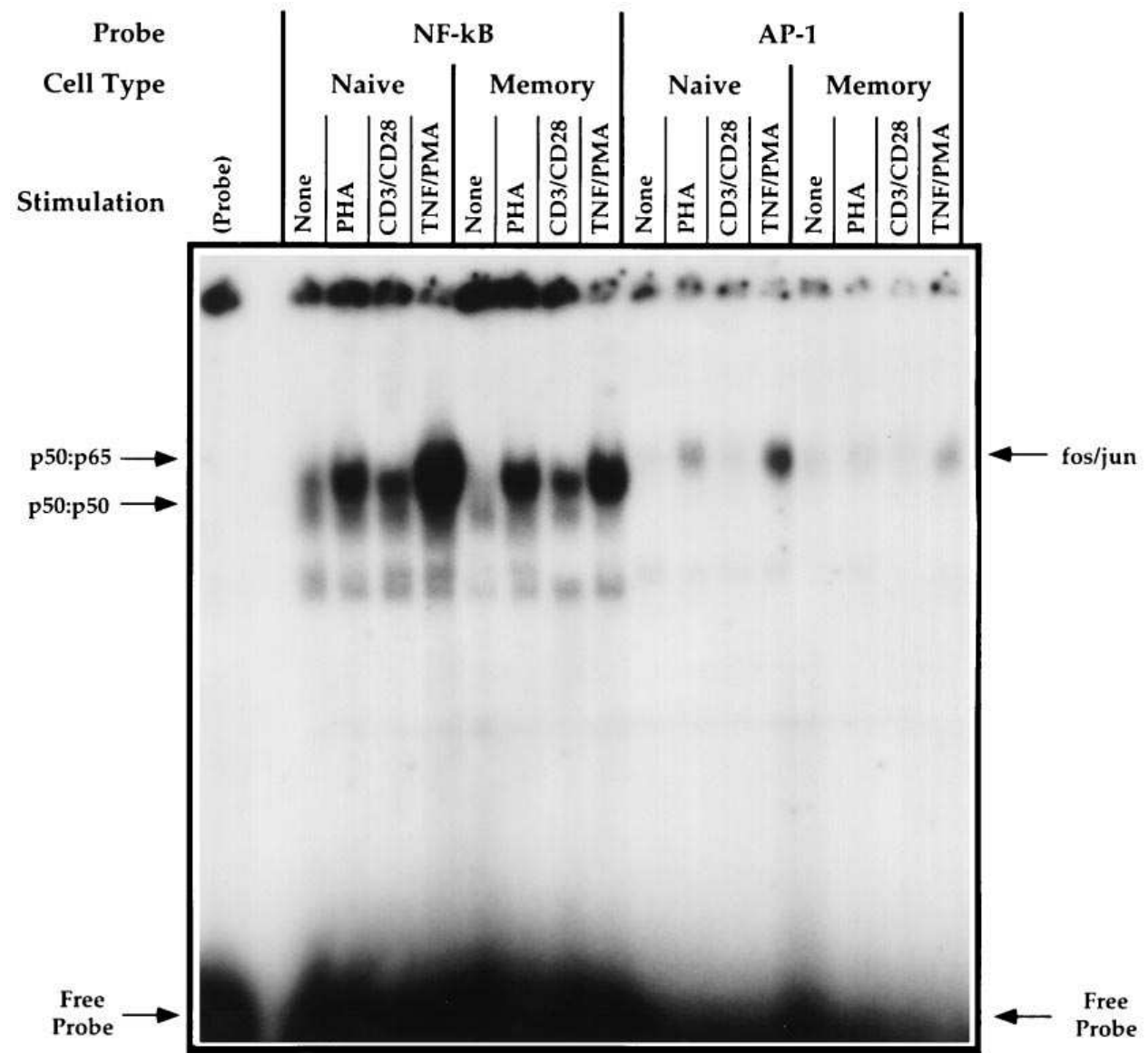

Figure 6. Naive and memory cells show similar activation of NF- $\mathrm{kB}$ and AP-1. Sorted cells were stimulated for $8 \mathrm{~h}$ with PHA or CD3/ CD28. Nuclear extracts were prepared and assayed for $\mathrm{NF}-\kappa \mathrm{B}$ or AP-1 binding activity by electrophoretic mobility shift assay. Arrows show the bands corresponding to p50:p50 homodimers and p50:p65 heterodimers $(N F-\kappa B)$, or the AP-1 binding complex.

(naive T cells stimulated with $\mathrm{CD} 3 / \mathrm{CD} 28$ ). Even when stimulated with PHA, memory cells, which had significantly lower proliferative responses than naive $\mathrm{T}$ cells, still produced as much or more virus.

These findings have importance for the interpretation of data from viral replication or other studies with cultured PBMC, since the proportions of various subsets in these cultures is uncontrolled and usually unknown. As Fig. $2 E$ shows, the amount of viral replication obtained from a mixture of CD4 T cells is primarily dependent on the representation of memory cells in the culture. Similarly, results from functional assays (e.g., proliferation or cytokine production) will be heavily influenced by the memory cell representation in the culture, since memory $\mathrm{T}$ cells have very different functional capacities. While the representation of naive cells in the periphery averages $50 \%$ in healthy individuals (7), there is still considerable variation. Thus, comparison of functional data including HIV replication between different donors must be done in the context of the underlying composition of the cell types.

This problem is even greater in samples from HIV-infected individuals. These individuals, especially in late-stage disease, usually have significantly decreased representation of the naive CD4 T cells, and consequently increased proportions of memory $\mathrm{T}$ cells (7). Conclusions concerning functional differences in PBMC between HIV-infected and healthy individuals should be evaluated with these changes in mind to assess the impact of cell subset representation changes on the results. Indeed, functional changes that have been demonstrated in bulk populations may be due solely to subset representation, with no change whatsoever in the functional capacity of any given cell type.

Our experiments were performed using a T cell tropic virus (LAI); it remains to be determined whether or not similar observations will hold true for monocytotropic viruses. Certainly, tropism may affect the ability of different viruses to enter different subsets, even subsets of T cells. Such an effect would be amplified in long term cultures, where multiple cycles of reinfection can occur. In addition, monocytotropic viruses may not show the same inhibition for replication once they have entered naive T cells as does LAI.

The data we have presented here extend the demonstration that naive T cells are intrinsically resistant to HIV (4). Clearly, HIV is capable of infecting these cells, but this infection is rendered nonproductive when the cells are stimulated with the physiological signals provided by CD3/CD28 crosslinking. (Importantly, the inhibition demonstrated in our experiments is an effect on virus that has already entered cells. Other mechanisms, such as specific downregulation of coreceptors, cannot be ruled out in playing a role in the inhibition of virus in long term cultures such as those described by Levine et al.; reference 8.) As yet, we do not understand the basis for this inhibition. The naive $\mathrm{T}$ cells are responding strongly to the stimulation, including activating the nuclear factors NF- $\kappa \mathrm{B}$ and AP-1. It is possible that inhibition of HIV occurs at a pretranscriptional stage; i.e., steps of virus processing after infection, including integration. However, it is also possible that mechanisms leading to differential regulation of the HIV LTR is responsible. Certainly, naive T cells do not make most cyto- 
kines after $\mathrm{CD} 3 / \mathrm{CD} 28$ stimulation: thus, a negative regulatory mechanism exists in these cells to prevent expression of those cytokine genes. Since regulation of the HIV LTR is in many ways similar to that of inflammatory genes, it is quite possible that the HIV LTR is negatively regulated by the same mechanisms.

On the basis of these results, we have begun to assess the amount of HIV replication that is occurring in these subsets in vivo. Preliminary results suggest that, in fact, naive T cells are completely devoid of HIV in vivo (M. Roederer, manuscript in preparation). Combined with the data presented here, this provides a mechanism for the observation that $\mathrm{CD} 3 / \mathrm{CD} 28$-stimulated ex vivo expansion of CD4 T cells from HIV-infected adults leads to eradication of virus from the cultures (8). Specifically: CD3/CD28 stimulation preferentially expands naive $\mathrm{T}$ cells (since they have a greater proliferative response); the relative lack of virus in these cells combined with their ability to suppress extant virus leads to progressive dilution of HIV.

Levine et al. suggested that the CD3/CD28 ex vivo expansion of CD4 T cells might be useful as an immune-based cell therapy for HIV disease (8). Our data supports their observations and suggestion. Indeed, the selective expansion of naive $\mathrm{T}$ cells, the population of cells prerequisite for novel immune responses, is desirable for immunological restoration in immunodeficient patients.

Finally, the data that naive $\mathrm{T}$ cells are resistant to HIV in vitro and are uninfected in vivo rules out direct killing by HIV as the mechanism accounting for the preferential loss of this subset of CD4 T cells during the progression of HIV disease (7). This is not surprising, since naive CD8 T cells are lost at the same rate as naive CD4 T cells (7). Together, these data support a model in which thymopoiesis is progressively (or absolutely) destroyed early in HIV disease. Thus, long term therapy of HIV disease should include strategies aimed at restoring thymopoiesis.

Woods et al. (31) report that naive, but not memory, CD4 $\mathrm{T}$ cells show a loss in inducible virus after infection by HIV. While using a substantially different stimulation from ours (i.e., a monokine supernatant), their findings are in agreement with the conclusions of our paper. Together, our reports provide strong evidence that naive CD4 T cells are inherently resistant to productive HIV infection after physiological stimulation.

\section{Acknowledgments}

We thank Dr. Thomas Schall for collaboration on the chemokineinduced calcium flux experiments, Tatiana Auer for performing the quantitative PCR assays for HIV, and Dr. Ned Landau for sharing data on chemokine receptor expression. We are grateful to Dr. Thomas Merigan for making available the facilities of the Stanford Center for AIDS Research facility. We thank Drs. Peter Katsikis and Rachel Gerstein for critical reading of the manuscript, and other members of the Herzenberg laboratory for advice and help, and to Dr. David Parks and the Stanford Shared Fluorescence Activated Cell Sorter Facility for expert flow cytometry support.

This work was supported by National Institutes of Health grants CA-42509, AI-31770, and LM-04836.

\section{References}

1. Sanders, M.E., M.W. Makgoba, and S. Shaw. 1988. Human naive and memory T cells. Immunol. Today. 9:195-199.

2. Picker, L., J. Treer, D. Ferguson, P. Collins, D. Buck, and L. Terstappen.
1993. Control of lymphocyte recirculation in man. I. Differential regulation of the peripheral lymph node homing receptor L-selectin on T cells during the virgin to memory cell transition. J. Immunol. 150:1105-1121.

3. Okumura, M., Y. Fujii, K. Takeuchi, K. Inada, K. Nakahara, and H. Matsuda. 1993. Age-related accumulation of LFA-1 $1^{\text {high }}$ cells in a CD8+CD45RA ${ }^{\text {high }}$ T cell population. Eur. J. Immunol. 23:1057-1063.

4. Schnittman, S., H. Lane, J. Greenhouse, J. Justement, M. Baseler, and A. Fauci. 1990. Preferential infection of CD4+ memory T cells by human immunodeficiency virus type 1: evidence for a role in the selective T-cell functional defects observed in individuals. Proc. Natl. Acad. Sci. USA. 87:6058-6062.

5. Cayota, A., F. Vuillier, F. Scott-Algara, and G. Dighiero. 1990. Preferential replication of HIV-1 in the memory CD4+ subpopulation in asymptomatic HIV-seropositive patients. Lancet (N. Am. Ed.). 336:941-942.

6. Cayota, A., F. Vuillier, D. Scott-Algara, V. Feuillie, and G. Dighiero. 1993. Differential requirements for HIV-1 replication in naive and memory CD4 T cells from asymptomatic HIV-1 seropositive carriers and AIDS patients. Clin. Exp. Immunol. 91:241-248.

7. Roederer, M., J.G. Dubs, M.T. Anderson, P.A. Raju, L.A. Herzenberg, and L.A. Herzenberg. 1995. CD8 naive T cell counts decrease progressively in HIV-infected adults. J. Clin. Invest. 95:2061-2066.

8. Levine, B., J.D. Mosca, J.L. Riley, R.G. Carroll, M.T. Vahey, L.L. Jagodzinski, K.F. Wagner, D.L. Mayers, D.S. Burke, O.S. Weislow, et al. 1996. Antiviral effect and ex vivo CD4+ T cell proliferation in HIV-positive patients as a result of CD28 costimulation. Science (Wash. DC). 272:1939-1943.

9. Azuma, M., M. Cayabyab, D. Buck, J.H. Phillips, and L.L. Lanier. 1993. CD28 interaction with B7 co-stimulates primary allogeneic proliferative responses and cytotoxicity mediated by small, resting T lymphocytes. $J$. Exp. Med. 175:353.

10. Schmid, I., C.H. Uittenbogaart, and J.V. Giorgi. 1994. Sensitive method for measuring apoptosis and cell surface phenotype in human thymocytes by flow cytometry. Cytometry. 15:12-20.

11. Folks, T.M., K.A. Clouse, J. Justement, A. Rabson, E. Duh, J.H. Kehrl, and A.S. Fauci. 1989. Tumor necrosis factor alpha induces expression of human immunodeficiency virus in a chronically infected T-cell clone. Proc. Natl. Acad. Sci. USA. 86:2365-2368.

12. Raju, P.A., L.A. Herzenberg, L.A. Herzenberg, and M. Roederer. 1994. Glutathione precursor and antioxidant activities of $\mathrm{N}$-acetylcysteine and oxathiazolidine carboxylate compared in in vitro studies of HIV replication. AIDS Res. Hum. Retroviruses. 10:961-967.

13. Piatak, M., Jr., K.C. Luk, B. Williams, and J.D. Lifson. 1993. Quantitative competitive polymerase chain reaction for accurate quantitation of HIV DNA and RNA species. Biotechniques. 14:70-81.

14. Mulder, J., N. McKinney, C. Christopherson, J. Sninsky, L. Greenfield, and S. Kwok. 1994. Rapid and simple PCR assay for quantitation of human immunodeficiency virus type 1 RNA in plasma: application to acute retroviral infection. J. Clin. Microbiol. 32:292-300.

15. Roederer, M., F.J.T. Staal, P.A. Raju, S.W. Ela, L.A. Herzenberg, and L.A. Herzenberg. 1990. Cytokine-stimulated human immunodeficiency virus replication is inhibited by $N$-acetyl-L-cysteine. Proc. Natl. Acad. Sci. USA. 87: 4884-4888.

16. Roederer, M., S. Fiering, and L.A. Herzenberg. 1991. FACS-Gal: flow cytometric analysis and sorting of cells expressing reporter gene constructs. Methods (Orlando). 2:248-260.

17. Fried, M., and D.M. Crothers. 1981. Equilibria and kinetics of lac repressor-operator interactions by polyacrylamide gel electrophoresis. Nucleic Acids Res. 9:6505-6525.

18. Garner, M.M., and A. Revzin. 1981. A gel electrophoresis method for quantifying the binding proteins to specific DNA regions: application to components of the Escherichia coli lactose operon system. Nucleic Acid Res. 9: 3047-3060.

19. Roederer, M., M. Bigos, T. Nozaki, R.T. Stovel, D.R. Parks, and L.A. Herzenberg. 1995. Heterogeneous calcium flux in peripheral T cell subsets revealed by five-color flow cytometry using log-ratio circuitry. Cytometry. 21:187-196.

20. Nabel, G., and D. Baltimore. 1987. An inducible transcription factor activates expression of human immunodeficiency virus in T cells. Nature (Lond.). 326:711-713.

21. Feng, Y., C.C. Broder, P.E. Kennedy, and E.A. Berger. 1996. HIV-1 entry cofactor: functional cDNA cloning of a seven-transmembrane, $\mathrm{G}$ proteincoupled receptor. Science (Wash. DC). 272:872-877.

22. Deng, H., R. Liu, W. Ellmeier, S. Choe, D. Unutmaz, M. Burkhart, P.D. Marzio, S. Marmon, R.E. Sutton, C.M. Hill, et al. 1996. Identification of a major co-receptor for primary isolates of HIV-1. Nature (Lond.). 381:661-666.

23. Dragic, T., V. Litwin, G.P. Allaway, S.R. Martin, Y. Huang, K.A. Nagashima, C. Cayana, P.J. Maddon, R.A. Koup, J.P. Moore, and W.A. Paxton 1996. HIV-1 entry into CD4+ cells is mediated by the chemokine receptor CCCKR-5. Nature (Lond.). 381:667-673.

24. Alkhatib, G., C. Combadiere, C.C. Broder, Y. Feng, P.E. Kennedy, P.M. Murphy, and E.A. Berger. 1996. CC CKR5: A RANTES, MIP-1 $\alpha$, MIP-1 $\beta$ receptor as a fusion cofactor for macrophage-tropic HIV-1. Science (Wash. DC). 272:1955-1958.

25. Doranz, B.J., J. Rucker, Y. Yi, R.J. Smyth, M. Samson, S.C. Peiper, M. Parmentier, R.G. Collman, and R.W. Doms. 1996. A dual-tropic primary HIV-1 
isolate that uses fusin and the beta-chemokine receptors CKR-5, CKR-3, and CKR-2b as fusion cofactors. Cell. 85:1149-1158.

26. Choe, H., M. Farzan, Y. Sun, N. Sullivan, B. Rollins, P.D. Ponath, L. Wu, C.R. Mackay, G. LaRosa, et al. 1996. The beta-chemokine receptors CCR3 and CCR5 facilitate infection by primary HIV-1 isolates. Cell. 85:1135-1148.

27. Bleul, C.C., M. Farzan, H. Choe, C. Parolin, I. Clarklewis, J. Sodroski, and T.A. Springer. 1996. The lymphocyte chemoattractant SDF-1 is a ligand for LESTR/fusin and blocks HIV-1 entry. Nature (Lond.). 382:829-833.

28. Oberlin, E., A. Amara, F. Bachelerie, C. Bessia, J.L. Virelizier, F. Arenzanaseisdedos, O. Schwartz, J.M. Heard, I. Clarklewis, D.F. Legler, et al. 1996. The CXC chemokine SDF-1 is the ligand for LESTR/fusin and prevents infection by T-cell-line-adapted HIV-1. Nature (Lond.). 382:833-835.
29. Kobayashi, N., Y. Hamamoto, N. Yamamoto, A. Ishii, M. Yonehara, and S. Yonehara. 1990. Anti-Fas monoclonal antibody is cytocidal to human immunodeficiency virus-infected cells without augmenting viral replication. Proc. Natl. Acad. Sci. USA. 87:9620-9624.

30. Roederer, M., P.A. Raju, F.J.T. Staal, L.A. Herzenberg, and L.A. Herzenberg. 1991. N-Acetylcysteine inhibits latent HIV expression in chronically infected cells. AIDS Res. Hum. Retroviruses. 7:563-567.

31. Woods, T., B.D. Roberts, S.T. Butera, and T.M. Folks. 1997. Loss of inducible virus in CD45RA naive cells after human immunodeficiency virus-1 entry accounts for preferential viral replication in CD45RO memory cells. Blood. $89: 1635-1642$ 